\title{
Exercício físico e o controle da pressão arterial $^{*}$
}

\author{
Maria de Fátima Monteiro e Dário C. Sobral Filho²
}

\section{RESUMO}

O exercício físico provoca uma série de respostas fisiológicas, resultantes de adaptações autonômicas e hemodinâmicas que vão influenciar o sistema cardiovascular. Diversos estudos demonstraram o seu efeito benéfico sobre a pressão arterial. Sendo a hipertensão arterial sistêmica uma entidade de alta prevalência e elevada morbimortalidade na população, o exercício físico tem importante papel como elemento não medicamentoso para o seu controle ou como adjuvante ao tratamento farmacológico.

\section{RESUMEN}

\section{Ejercício físico y el control de la presion arterial}

El ejercicio físico provoca una serie de respuesta fisiológicas, resultantes de adaptaciones autonómicas y hemodinámicas que van a influenciar en el sistema cardiovascular. Diversos estudios demonstraron el efecto benéfico sobre la presión arterial. Siendo así, la hipertensión arterial sistémica una entidad de alta prevalencia y elevada morbi-mortalidad en la población, el ejercicio físico tiene un papel muy importante como elemento no medicamentoso para su control y como adyuvante al tratamiento farmacológico tambien.

\section{INTRODUÇÃO}

O exercício físico é uma atividade realizada com repetições sistemáticas de movimentos orientados, com conseqüente aumento no consumo de oxigênio devido à solicitação muscular, gerando, portanto, trabalho(1). O exercício representa um subgrupo de atividade física planejada com a finalidade de manter o condicionamento ${ }^{(2)}$. Pode também ser definido como qualquer atividade muscular que gere força e interrompa a homeostase ${ }^{(3)}$.

O exercício físico provoca uma série de respostas fisiológicas nos sistemas corporais e, em especial, no sistema cardiovascular. Com o objetivo de manter a homeostasia celular em face do aumento das demandas metabólicas, alguns mecanismos são acionados $^{(4)}$. Esses mecanismos funcionam sob a forma de arcos reflexos constituídos de receptores, vias aferentes, centros integradores, vias eferentes e efetores; muitas etapas desses mecanismos ainda não foram completamente elucidadas ${ }^{(5)}$.

* Hospital Universitário Oswaldo Cruz - Universidade de Pernambuco. Rua Arnóbio Marques, 310 - 50100-130 - Recife, PE.

1. Especialista em Cardiologia (AMB-SBC) e habilitada em Ergometria (AMB-SBC). Ergometrista do Hospital Universitário Oswaldo Cruz - Universidade de Pernambuco.

2. Professor Adjunto de Cardiologia da Faculdade de Ciências Médicas Universidade de Pernambuco. Mestre em Medicina Interna e Doutor em Cardiologia.

Recebido em 3/9/04. 2a versão recebida em 10/11/04. Aceito em 25/11/04.

Endereço para correspondência: Maria de Fátima Monteiro, Rua Teles Júnior, 155-B, apto. 1.201 - 52050-040 - Recife, PE. E-mail: fatimam@cardiol.br
Palavras-chave: Atividade física. Hipertensão arterial. Tratamento não-farmacológico.

Palabras-clave: Atividad física. Hipertensión arterial. Tratamiento no farmacológico.

\section{EFEITOS FISIOLÓGICOS DO EXERCÍcIO}

Os mecanismos responsáveis pelos ajustes do sistema cardiovascular ao exercício e os índices de limitação da função cardiovascular constituem aspectos básicos relacionados ao entendimento das funções adaptativas. Esses mecanismos são multifatoriais e permitem ao sistema operar de maneira efetiva nas mais diversas circunstâncias. Os ajustes fisiológicos são feitos a partir das demandas metabólicas, cujas informações chegam ao tronco cerebral através de vias aferentes, até a formação reticular bulbar, onde se situam os neurônios reguladores centrais ${ }^{(1)}$.

Os efeitos fisiológicos do exercício físico podem ser classificados em agudos imediatos, agudos tardios e crônicos. Os efeitos agudos, denominados respostas, são os que acontecem em associação direta com a sessão de exercício; os efeitos agudos imediatos são os que ocorrem nos períodos peri e pós-imediato do exercício físico, como elevação da freqüência cardíaca, da ventilação pulmonar e sudorese; já os efeitos agudos tardios acontecem ao longo das primeiras 24 ou 48 horas (às vezes, até 72 horas) que se seguem a uma sessão de exercício e podem ser identificados na discreta redução dos níveis tensionais, especialmente nos hipertensos, na expansão do volume plasmático, na melhora da função endotelial|(4,5) e na potencialização da ação e aumento da sensibilidade insulínica na musculatura esquelética ${ }^{(5-7)}$. Por último, os efeitos crônicos, também denominados adaptações, resultam da exposição freqüente e regular às sessões de exercícios e representam aspectos morfofuncionais que diferenciam um indivíduo fisicamente treinado de outro sedentário, tendo como exemplos típicos a bradicardia relativa de repouso, a hipertrofia muscular, a hipertrofia ventricular esquerda fisiológica e o aumento do consumo máximo de oxigênio $\left(\dot{V}_{2} \text { máximo }\right)^{(4,5)}$. O exercício também é capaz de promover a angiogênese, aumentando o fluxo sanguíneo para os músculos esqueléticos e para o músculo cardíaco(3,8).

O exercício físico realizado regularmente provoca importantes adaptações autonômicas e hemodinâmicas que vão influenciar o sistema cardiovascular(7), com o objetivo de manter a homeostasia celular diante do incremento das demandas metabólicas. Há aumento no débito cardíaco, redistribuição no fluxo sanguíneo e elevação da perfusão circulatória para os músculos em atividade ${ }^{(4)}$. A pressão arterial sistólica (PAS) aumenta diretamente na proporção do aumento do débito cardíaco. A pressão arterial diastólica reflete a eficiência do mecanismo vasodilatador local dos músculos em atividade, que é tanto maior quanto maior for a densidade capilar local $^{(1,9)}$. A vasodilatação do músculo esquelético diminui a resistência periférica ao fluxo sanguíneo e a vasoconstrição concomitante que ocorre em tecidos não exercitados induzida simpaticamente compensa a vasodilatação. Conseqüentemente, a resistência total ao fluxo sanguíneo cai drasticamente quando o exercício começa, alcançando um mínimo ao redor de $75 \%$ do $\mathrm{V}_{2}$ máximo ${ }^{(3)}$. Os níveis tensionais elevam-se durante o exercício físico e no esforço predominantemente estático, tendo já sido constatados, em indivíduos jovens e saudáveis, níveis de pressão intra-arterial superiores a 400/250mmHg sem causar danos à saúde(4,10). 
Em resumo, pode-se dizer que durante um período de exercício, o corpo humano sofre adaptações cardiovasculares e respiratórias a fim de atender às demandas aumentadas dos músculos ativos e, à medida que essas adaptações são repetidas, ocorrem modificações nesses músculos, permitindo que o organismo melhore o seu desempenho. Entram em ação processos fisiológicos e metabólicos, otimizando a distribuição de oxigênio pelos tecidos em atividade $^{(11)}$. Portanto, os mecanismos que norteiam a queda pressórica pós-treinamento físico estão relacionados a fatores hemodinâmicos, humorais e neurais(12).

\section{EXERCÍCIO FÍSICO NO TRATAMENTO DA HIPERTENSÃO ARTERIAL SISTÊMICA}

A hipertensão arterial sistêmica representa uma das maiores causas de morbidade cardiovascular no Brasil e acomete 15\% a $20 \%$ da população adulta, possuindo também considerável prevalência em crianças e adolescentes. Considerada um dos principais fatores de risco de morbidade e mortalidade cardiovasculares, representa alto custo social, uma vez que é responsável por cerca de $40 \%$ dos casos de aposentadoria precoce e absenteísmo no trabalho em nosso meio ${ }^{(5,13)}$. A identificação e o tratamento de pacientes com hipertensão arterial sistêmica constituem um problema de saúde pública no Brasil.

O sedentarismo também constitui importante fator de risco, já estando bem estabelecida a ocorrência de maior taxa de eventos cardiovasculares e maior taxa de mortalidade em indivíduos com baixo nível de condicionamento físico ${ }^{(14)}$. Estima-se que a prevalência do sedentarismo seja de até $56 \%$ nas mulheres e $37 \%$ nos homens, na população urbana brasileira(15).

Modificações no estilo de vida, incluindo exercício físico, são recomendadas no tratamento da hipertensão arterial. Estudo envolvendo 217 pacientes de ambos os sexos, com idade variando de 35 a 83 anos, mostrou que a adesão a medidas não farmacológicas, dentre as quais a prática de exercício físico, promoveu sensível efeito na redução dos níveis pressóricos ${ }^{(16)}$

Estudos randomizados mostraram efeitos indesejáveis do tratamento farmacológico em subgrupos de pacientes com hipertensão arterial sistêmica, sugerindo uma mudança na abordagem do tratamento dos mesmos. O efeito do exercício físico sobre os níveis de repouso da pressão arterial de grau leve a moderado é especialmente importante, uma vez que o paciente hipertenso pode diminuir a dosagem dos seus medicamentos anti-hipertensivos ou até ter a sua pressão arterial controlada, sem a adoção de medidas farmacológicas ${ }^{(7,15)}$. A tendência de utilizar precocemente agentes farmacológicos foi substituída por agentes não farmacológicos, dentre estes, o exercício físico aeróbico tem sido recomendado para o tratamento da hipertensão arterial sistêmica leve ${ }^{(15)}$. Todavia, somente $75 \%$ dos pacientes hipertensos são responsivos ao treinamento físico, uma vez que a hipertensão arterial sistêmica é uma síndrome poligênica e que pode ser influenciada pela herança genética $^{(7)}$.

Paffenbarger et al.(17), em um seguimento de seis a 10 anos, de 15.000 indivíduos diplomados de Harvard, constataram que os que praticavam exercício físico de forma regular apresentavam risco $35 \%$ menor de desenvolver hipertensão arterial do que os indivíduos sedentários.

Em outro estudo, Paffenbarger et al.(18) seguiram 10.260 diplomados de Harvard por mais de uma década e constataram uma relação inversa entre a prática de atividade física e o risco de morte por todas as causas. Ao comparar os que iniciaram atividade esportiva moderadamente vigorosa (a uma intensidade de 4,5 ou mais equivalentes metabólicos) com aqueles que não participaram desse tipo de treinamento, observaram redução de $23 \%$ no risco de morte (intervalo de confiança de $95 \%, 4 \%$ para $42 \% ; p=0,015$ ). A mortalidade diminuiu mesmo em indivíduos de meia-idade que aumentaram a atividade física através de simples modificações nas atividades ocupacionais ou mesmo recreativas.

A busca de uma explicação para o efeito redutor do exercício sobre a pressão arterial de indivíduos normotensos e, principalmente, hipertensos tem motivado inúmeras pesquisas nas últimas décadas, sendo a redução da pressão arterial diastólica em repouso após treinamento a mais largamente estudada. Os mecanismos que norteiam a queda pressórica pós-treinamento físico estão relacionados a fatores hemodinâmicos, humorais e neurais ${ }^{(12)}$.

Dentre os fatores hemodinâmicos verificou-se, tanto em ratos espontaneamente hipertensos quanto em humanos, que o exercício físico promove redução da pressão arterial por diminuição no débito cardíaco que está associada ao decréscimo da freqüência cardíaca, uma vez que não foram observadas alterações no volume sistólico ${ }^{(12,19)}$. A queda na resistência vascular sistêmica e, conseqüentemente, na pressão arterial seria outro mecanismo alternativo proposto para explicar a queda na pressão arterial pós-exercício(12,19,20). Uma redução significativa nos níveis pressóricos é conseguida com treinamento de baixa intensidade $150 \%$ do consumo de oxigênio de pico). Assim, o exercício físico de baixa intensidade diminui a pressão arterial porque provoca redução no débito cardíaco, o que pode ser explicado pela diminuição na freqüência cardíaca de repouso e diminuição do tônus simpático no coração, em decorrência de menor intensificação simpática e maior retirada vagal|12,21,22).

Alguns autores atribuem a redução da pressão arterial após exercício físico em hipertensos a alterações humorais relacionadas à produção de substâncias vasoativas, como o peptídeo natriurético atrial ou ouabaína-like, modulada centralmente ${ }^{(12)}$. Ocorre, também, melhora na sensibilidade à insulina ${ }^{(6,7,23)}$, além da redução da noradrenalina plasmática, sugerindo redução da atividade nervosa simpática, associada ao aumento da taurina sérica e prostaglandina $E$, que inibem a liberação de noradrenalina nas terminações nervosas simpáticas e redução do fator ouabaína-like, que provocaria recaptação de noradrenalina nas fendas sinápticas ${ }^{(12)}$. Essa hipótese é contestada, uma vez que pode ser demonstrada redução da pressão arterial mesmo antes de haver redução nos níveis de noradrenalina plasmáticos. Outros autores relatam que os níveis de noradrenalina diminuem com o treinamento apenas nos indivíduos hiperadrenérgicos. Redução nos níveis da renina plasmática também foi verificada, bem como aumento na produção de ácido nítri$\mathrm{co}^{(6,12,24,25)}$.

O treinamento aeróbico por exercícios predominantemente isotônicos ou dinâmicos geralmente não modifica, nos normotensos, os níveis de pressão arterial sistólica e diastólica em repouso, embora a pressão arterial média possa declinar em função da menor freqüência cardíaca basal, após período de treinamento físi$\mathrm{co}^{(6)}$.

Grassi et al.(26) estudaram jovens normotensos e constataram que após 10 semanas de exercício físico, além de diminuição na pressão arterial sistólica e diastólica, houve redução significativa na atividade nervosa simpática (36\%), fato não observado no grupo controle, que não realizou exercício físico(12).

No que diz respeito ao efeito agudo do exercício sobre a curva da pressão arterial nas 24 horas em pacientes avaliados através da monitorização ambulatorial da pressão arterial (MAPA), Marceau et al. demonstraram que indivíduos treinados, a $50 \%$ e a $70 \%$ do $\dot{\mathrm{V}}_{2}$ máximo, apresentam diferentes perfis de curva pressórica; os treinados a $50 \%$ mantiveram a redução exclusivamente durante o período de vigília e os que treinaram a $70 \%$ mantiveram a redução durante o sono(27).

Ishikawa et al.(28) estudaram 109 indivíduos hipertensos nos estágios I e II que realizaram treinamento leve por oito semanas, em academias. Constataram que houve redução significativa da pressão arterial em todos eles; os indivíduos idosos apresentaram menor redução nos níveis pressóricos do que os indivíduos jovens. Não foi observada influência do sexo nos resultados. 
Mais recentemente, Takata et al. submeteram 207 indivíduos com hipertensão essencial de graus 1 e 2 a um programa de exercício físico por oito semanas. Foram divididos em cinco grupos baseados na duração e freqüência por semana de exercício (grupo controle - sedentários, 30 a 60 minutos/semana, 61 a 90, 91 a 120 e acima de 120 minutos/semana). Verificaram que a PA diastólica não mudou em repouso no grupo controle; no entanto, houve significativa redução na pressão arterial sistólica e diastólica em repouso nos quatro grupos submetidos a exercícios. A magnitude de redução na pressão arterial sistólica foi maior no grupo de 60 a 90 minutos/semana, comparada com o grupo de 30 a 60 minutos/ semana. Não houve redução maior com o aumento do volume de exercício. A magnitude de redução da pressão arterial diastólica não foi significativamente diferente nos quatro grupos. Não houve relação óbvia entre a freqüência de exercícios por semana e a magnitude de decréscimo dos níveis pressóricos provocado pelos exercícios ${ }^{(29)}$.

Indivíduos hipertensos mantêm a redução mais intensa da pressão arterial nas 24 horas seguintes às do exercício( ${ }^{(9)}$. Verificações da pressão arterial em sessões de 25 e 45 minutos após exercício físico, a $50 \%$ do consumo máximo de oxigênio, mostraram reduções mais acentuadas após as sessões de 45 minutos ${ }^{(30)}$. É possível que a queda da pressão arterial nesse caso se deva à diminuição na resistência vascular periférica ${ }^{20,24,31)}$, podendo ainda estar relacionada à vasodilatação provocada pelo exercício físico nas musculaturas ativa e inativa, resultante do acúmulo de metabólitos musculares provocado pelo exercício (potássio, lactato e adenosina) ou à dissipação do calor produzida pelo exercício físico ${ }^{(30,32)}$. Alternativamente, o aumento do fluxo sanguíneo pode decorrer da redução do tônus simpático e o conseqüente acréscimo da vasodilatação periférica(32), que parece estar relacionada à elevação da secreção de opióides endógenos provocada pelo exercício e que possuem efeito vasodilatador direto ${ }^{(30)}$.

Alterações funcionais dos pressorreceptores arteriais e cardiopulmonares, como o aumento na sua sensibilidade e modificação no seu ponto de ativação e do tempo de recuperação, podem também contribuir para o efeito vasodilatador pós-exercício. A redução na resposta vasoconstritora alfa-adrenérgica, verificada no período de recuperação - down-regulation dos receptores alfa-adrenérgicos também poderia explicar o maior fluxo sanguíneo muscular pós-exercício. E, ainda, fatores humorais como a adrenalina, o fator atrial natriurético e o óxido nítrico têm sido citados como fatores envolvidos na vasodilatação pós-exercício ${ }^{(9,30)}$.

Surpreendentemente, estudos com ratos espontaneamente hipertensos mostraram que a diminuição da resistência vascular periférica total não era o mecanismo hemodinâmico responsável pela diminuição da pressão arterial, após treinamento de baixa intensidade e, sim, uma redução no débito cardíaco(22). No estudo de Overton et al.(31), medições do fluxo sanguíneo, através do doppler, nas artérias ilíaca, mesentérica superior e renal de ratos, geneticamente hipertensos, treinados a $60 \%$ e $70 \%$ do $\mathrm{VO}_{2}$ máximo, não mostraram diminuição na resistência vascular regional em nenhuma das artérias, sugerindo que a redução do débito cardíaco seja o mecanismo responsável.

\section{RECOMENDAÇÕES PARA A PRÁTICA DO EXERCÍCIO NA HIPERTENSÃO ARTERIAL}

A Sociedade Brasileira de Cardiologia recomenda que os indivíduos hipertensos iniciem programas de exercício físico regular, desde que submetidos à avaliação clínica prévia. Os exercícios devem ser de intensidade moderada, de três a seis vezes por semana, em sessões de 30 a 60 minutos de duração, realizados com freqüência cardíaca entre $60 \%$ e $80 \%$ da máxima ou entre $50 \%$ e $70 \%$ do consumo máximo de oxigênio(33).

Com discretas modificações, essas recomendações estão concordantes com as de outras entidades internacionais ${ }^{(34,35)}$.

\section{CONCLUSÕES}

Conclui-se que os efeitos benéficos do exercício físico devem ser aproveitados no tratamento inicial do indivíduo hipertenso, visando evitar o uso ou reduzir o número de medicamentos e de suas doses. Em indivíduos sedentários e hipertensos, reduções clinicamente significativas na pressão arterial podem ser conseguidas com o aumento relativamente modesto na atividade física, acima dos níveis dos sedentários, além do que o volume de exercício requerido para reduzir a pressão arterial pode ser relativamente pequeno, possível de ser atingido mesmo por indivíduos sedentários.

Todos os autores declararam não haver qualquer potencial conflito de interesses referente a este artigo.

\section{REFERÊNCIAS}

1. Barros Neto TL, César MC, Tebexreni AS. Fisiologia do exercício. In: Ghorayeb N, Barros TL, editores. O exercício. Preparação fisiológica, avaliação médica, aspectos especiais e preventivos. São Paulo: Atheneu, 1999;3-13.

2. Wilmore JH, Costill DL. Controle cardiovascular durante o exercício. Fisiologia do esporte e do exercício. 2a ed. São Paulo: Manole, 2003.

3. Silverthorn DU. Fisiologia integrada. Fisiologia humana. Uma abordagem integrada. 2a ed. Barueri (SP): Manole, 2003.

4. Araújo CGS. Fisiologia do exercício físico e hipertensão arterial. Uma breve introdução. Revista Hipertensão, 2001;4. Disponível em: URL: http//www.sbh. org.br/revista_N3_V4. Acesso em 11 maio 2003.

5. I Consenso Nacional de Reabilitação Cardiovascular. Arq Bras Cardiol 1997;69 (4).

6. Arpad AA, Mastrocolla LE, Bertolami MC. Atuação do exercício físico sobre os fatores de risco para doenças cardiovasculares. Rev Socesp 1996;6:1-5.

7. Rondon MUPB, Brum PC. Exercício físico como tratamento não farmacológico da hipertensão arterial. Rev Bras Hipertens 2003;10:134-7.

8. Irigoyen MC, Angelis K D, Schaan BDA, Fiorino P, Michelini LC. Exercício físico no diabetes melito associado à hipertensão arterial sistêmica. Rev Bras Hipertens 2003;10:109-17.

9. Pássaro LC, Godoy M. Reabilitação cardiovascular na hipertensão arterial. Rev Socesp 1996;6:45-58.

10. Forjaz CLM, Rezk C, Melo CMM, Santos DA, Teixeira L, Nery SS, et al. Exercício resistido para o paciente hipertenso: indicação ou contra-indicação. Rev Bras Hipertens 2003;10:119-24.

11. Wilmore JH, Costill DL. Controle cardiovascular durante o exercício. In: Fisiologia do esporte e do exercício. 2a ed. São Paulo: Manole, 2003.

12. Negrão CE, Rondon MUPB, Kuniyosh FHS, Lima EG. Aspectos do treinamento físico na prevenção da hipertensão arterial. Revista Hipertensão, 2001;4. Disponível em URL: http//www.sbh.org.br/revista/2001_2001_V4. Acesso em 11 maio 2003.

13. III Consenso Brasileiro de Hipertensão Arterial - CBHA. Campos do Jordão, SP, 12 a 15 de fev., 1998 - SBC.

14. Myers J. Exercise and cardiovascular health. Circulation 2003;107:e2-e5.

15. Fuchs FD, Moreira DM, Ribeiro JP. Eficácia anti-hipertensiva do condicionamento físico aeróbio. Uma análise crítica das evidências experimentais. Arq Bras Cardiol 1993;61:187-90.

16. Ferreira KVS, Melo AMCA, Sobral Filho DC, Arruda IKG, Diniz AS, Toscano CHH. Arq Bras Cardiol 1999;73(Supl IV):110.

17. Paffenbarger RS, Wing AL, Hyde RT. Physical exercise and incidence of hypertension in college alumni. Am J Epidemiol 1983;117:245-57.

18. Paffenbarger RS Jr, Hyde RT, Wing AL, Lee IM, Jung DL, Kampert JE. The association of changes in physical-activity level and other lifestyle characteristics with mortality among men. N Engl J Med 1993;328:538-45.

19. Nami R, Mondillo S, Agricola E, Lenti S, Ferro G, Nami N, et al. Aerobic exercise training fails to reduce blood pressure in nondipper-type hypertension. Am J Hypertens 2000;13:593-600.

20. Jacopo M, Legramante JM, Galante, et al. A hemodynamic and autonomic correlates of post exercise hypotension in patients with mild hypertension. Am J Physiol Regul Integr Comp Physiol 2002;282:1037-43.

21. Teixeira JAC. Hipertensão arterial sistêmica e atividade física. Rev Socerj 2000; 13:25-30. 
22. Negrão CE, Rondon MUPB. Exercício físico, hipertensão e controle barorreflexo da pressão arterial. Rev Bras Hipertens 2001;8:89-95

23. Saraiva JF, Atique G. Exercício físico e hipertensão arterial. Relato de caso. Revista Hipertensão 2001;4. Disponível em URL: http://www.sbh.org.br/revista/ 2001_N3_V4. Acesso em 11 maio 2003.

24. Arrol B, Beaglehole R. Does physical activity lower blood pressure: a critical review of the clinical trials. J Clin Epidemiol 1992;5:439-47.

25. Barros Neto TL. Fisiologia do exercício aplicada ao sistema cardiovascular. Rev Socesp 1996;6:6-10

26. Grassi G, Seravalle G, Calhoun DA, Mancia G. Physical training and baroreceptor control of sympathetic nerve activity in humans. Hypertension 1994;23:294-301.

27. Marceau M, Kouame N, Lacourciere Y, Cleroux J. Blood pressure. Effects of different training intensities on 24-hour blood pressure in hypertensive subjects. Circulation 1993;88:2803-11.

28. Ishikawa K, Ohta T, Zhang J, Hashimoto S, Tanaka H. Influence of age and gender on exercise training-induced blood pressure reduction in systemic hypertension. Am J Cardiol 1999;84:192-6.
29. Takata KI, Ohta T, Tanaka H. How much exercise is required to reduce blood pressure in essential hypertensives: a dose-response study. Am J Hypertens 2003; 13:593-600

30. Forjaz CLM, Santaella DF, Rezende LO, Barreto ACP, Negrão CE. Duração do exercício determina a magnitude e a duração da hipotensão pós-exercício. Arq Bras Cardiol 1998;70:99-104.

31. Overton MJ, Joyner MJ, Tipton CM. Reductions in blood pressure after acute exercise by hypertensive rats. J Appl Physiol 1998;64:748-52.

32. Brownley K, Hinderliter AL, West SG, Girdler SS, Sherwod A, Light K. Sympathoadrenergic mechanisms in reduce hemodynamic stress response after exercise. Med Sci Sports Exerc 2003;35:978-86.

33. IV Diretrizes Brasileiras de Hipertensão 2002; cap. 5:13-14 - SBC.

34. ACC/AHA Guidelines for exercise testing: a report of the American College of Cardiology/American Heart Association Task Force on Practice Guidelines (Committee on Exercise Testing). J Am Coll Cardiol 30:260-315.

35. Cléroux J, Feldman DR, Petrella RJ. Recommendations on physical exercise training. J Can Med Assoc 1999;160(Suppl 9):S21-8. 